\title{
Effect of Sodium Cromoglycate on Acetic Acid-induced Ulcerative Colitis in Mice
}

\author{
Zahra Ghafouri, Saeid Seyyedian", Jafar Nikbakht², Ebrahim Kouhsari ${ }^{3,4}$, Sara Bayat ${ }^{1}$, \\ Hamidreza Zargar ${ }^{5}$ and Gholamreza Houshmand ${ }^{6,7}$
}

Department of Biochemistry, Biophysics and Genetics, School of Medicine, Mazandaran University of Medical Sciences, Sari; Department of Internal Medicine, School of Medicine, Alimentary Tract Research Center, Ahvaz Jundishapur University of Medical Sciences ${ }^{1}$, Ahvaz; Department of Pharmacology, School of Medicine, Yasuj University of Medical Science ${ }^{2}$, Yasuj; Clinical Microbiology Research Center, Ilam University of Medical Sciences ${ }^{3}$, Ilam; Laboratory Sciences Research Center, Golestan University of Medical Sciences ${ }^{4}$, Gorgan; Department of Pharmacology, School of Pharmacy, Ahvaz Jundishapur University of Medical Sciences ${ }^{5}$, Ahvaz; Department of Pharmacology, School of Medicine $^{6}$, Gut and Liver Research Center ${ }^{7}$, Mazandaran University of Medical Sciences, Sari, Iran

Background/Aims: Ulcerative colitis (UC) is a type of inflammatory bowel disease that mainly involves the colon. Thus far, glucocorticoids and amino-salicylate have been the main treatment.

Methods: To assess drugs with fewer side effects, this study evaluated the effects of sodium cromoglycate (SCG) on acetic acid-induced UC in rats. The treatment groups included SCG receivers (50 and $100 \mathrm{mg} / \mathrm{kg}$, intra-orally) and sulfasalazine (SSZ) receivers (100 mg/kg, intra-orally). The colonic mucosal injury was assessed by clinical, macroscopic, and histopathological examinations. Results: In the treatment groups with 50 and $100 \mathrm{mg} / \mathrm{kg}$ of SCG, the clinical activity score decreased to $2.67 \pm 0.18$ and $1.73 \pm 0.21$ $(p<0.05)$, respectively, compared to the UC control group $(3.21 \pm 0.31)$, and were higher than that of the group given the standard treatment of $100 \mathrm{mg} / \mathrm{kg} \mathrm{SSZ}$ (1.10 \pm 0.09 ). The treatment groups with 50 and $100 \mathrm{mg} / \mathrm{kg}$ of SCG showed a lower clinical gross lesion score than the UC control group $(2.91 \pm 0.28$ and $2.10 \pm 0.43$, vs. $4.49 \pm 0.61, p<0.05)$ and were higher than the standard group $(0.95 \pm 0.18)$. Treatment with SCG $(100 \mathrm{mg} / \mathrm{kg})$ decreased the macroscopic scores significantly compared to the UC control group $(p<0.05)$ on the 8th day.

Conclusions: SCG $(100 \mathrm{mg} / \mathrm{kg})$ decreased significantly the clinical activity score, gross lesion, and percentage-affected area compared to the UC controls on the 8th day. (Korean J Gastroenterol 2020;75:39-45)

Key Words: Sodium cromoglycate; Acetic acid; Colitis, ulcerative

\section{INTRODUCTION}

Inflammatory bowel disease (IBD) is a chronic immunological disease of the gastrointestinal tract associated with austere ulceration, relapse bleeding, and diarrhea. IBD consists of two sub-families: Crohn's disease and ulcerative colitis (UC). Crohn's disease might involve all lining sites of the digestive tract, while UC is more specific to some parts of the gas- trointestinal tract, mainly the colon. ${ }^{1} \mathrm{UC}$ is generally a T-helper cell type 2-driven response that primarily affects the colonic mucosa and sub-mucosa. ${ }^{2}$ The most common symptoms of UC are ulcers and inflammation of the inner lining of the colon that lead to bloody diarrhea, abdominal pain, urgency, and tenesmus. ${ }^{3}$ Oxidative stress has been implicated in the pathogenesis of UC. The excess production of reactive oxygen species, such as superoxide, hydroxyl radical, hydrogen per-

Received June 26, 2019. Revised November 10, 2019. Accepted November 23, 2019.

(C) This is an open access article distributed under the terms of the Creative Commons Attribution Non-Commercial License (http://creativecommons.org/licenses/ by-nc/4.0) which permits unrestricted non-commercial use, distribution, and reproduction in any medium, provided the original work is properly cited.

Copyright (c) 2020. Korean Society of Gastroenterology.

Correspondence to: Gholamreza Houshmand, Department of Pharmacology, School of Medicine, Mazandaran University of Medical Sciences, Payambar Azam Complex, 18th km of Farah Abad Blvd, Khazar Square, Sari, Mazandaran, Iran. Tel: +98-914343838, Fax: +98-1133543081, E-mail: dr.houshmand_pharmaco@yahoo.com, ORCID: https://orcid.org/0000-0001-8931-9613

Financial support: Mazandaran University of Medical Sciences.

Conflict of interest: None. 
oxide, hypochlorous acid, and oxidant derivatives of $\mathrm{N}$-chloramines, might be found in the inflamed mucosa of a UC case. ${ }^{4}$ Intestinal mucosal immune cells, such as mast cells and eosinophils, are crucial in the pathogenesis of UC. ${ }^{5}$ Chemically induced UC models are beneficial for studying the role of mast cells in the pathogenesis of the disease. Several chemical agents are employed to induce UC models, including trinitrobenzene sulfonic acid, dextran sodium sulfate, and acetic acid (AA). ${ }^{6}$ Thus far, glucocorticoids and amino-salicylate have been used for UC treatment, ${ }^{7}$ but their side effects remain a major clinical problem. ${ }^{8}$ Sodium cromoglycate (SCG) is an effective drug for the treatment of disorders associated with type I hypersensitivity reactions accompanied by mast cell degranulation and its importance in the management of asthma is accepted widely. ${ }^{9,10}$ As there is evidence of tissue eosinophilia and mast cell activity followed by increased histamine secretion in UC patients ${ }^{11}$ and a lower incidence of side effects by cromolyn administration compared to glucocorticoids and aminosalicylate, ${ }^{12}$ it was believed that SCG might be valuable in UC management. This study evaluated the impact of SCG on AA-induced UC through a com- parison of sulfasalazine (SSZ) treatment as a standard procedure.

\section{SUBJECTS AND METHODS}

\section{Animals}

All experimental procedures utilizing mice were performed in accordance with the National Institute of Health guidelines. Male (220-250 g, n=48) Kunming mice were obtained from the Experimental Animal Center, Yasuj University of Medical Science (Yasuj, Iran). They were maintained in an animal house with a controlled constant temperature of $23 \pm 2^{\circ} \mathrm{C}$, relative humidity of $55 \pm 5 \%$, and a 12 hours light/dark cycle. The animals were allowed access to tap water and standard laboratory chow ad libitum. The experiments were performed between 08:00 and 12:00 a.m. to avoid circadian influences.

\section{Induction of UC}

The animals were divided randomly into six groups consisting of eight members. Group I served as the normal control and received $1 \mathrm{~mL}$ saline intra-rectally after the administration of saline orally. Group II received $1 \mathrm{~mL}$ saline intra-rectally following the oral administration of SCG (100 $\mathrm{mg} / \mathrm{kg}$ ). Group III served as the UC control and received
$1 \mathrm{~mL}$ AA intra-rectally following the oral administration of saline. Groups IV and V received $1 \mathrm{~mL}$ AA intra-rectally following the administration of SCG at 50 and $100 \mathrm{mg} / \mathrm{kg}$ doses, respectively. Group VI received $1 \mathrm{~mL}$ AA intra-rectally following the administration of SSZ (100 mg/kg, intra-orally). All of the oral treatments were given for 3 days using an oral gavage. On the 4th day of the treatment, the animals were fasted overnight with access to water ad libitum. On the 5th day, 1 hour after the oral treatment, the animals were anesthetized by ether inhalation, and a $2 \mathrm{~mm}$ diameter polypropylene tube was inserted through the rectum into the colon with a distance of $8 \mathrm{~cm}$. A solution of $1 \mathrm{~mL}$ of $\mathrm{AA}(5 \%$ in $0.9 \%$ saline in groups III, IV, V, and VI) was instilled into the lumen of the colon and maintained in a supine Trendelenburg position for 30 seconds to prevent leakage of the intra-colonic instillation. After 72 hours of single-dose administration of AA (8th day), the clinical activity scores were measured. The animals (half of each group) were sacrificed by a cervical dislocation and the colon was dissected. The colon was flushed gently with saline, weighed, and used for macroscopic scoring and histopathology estimations. The same procedure was performed in the remaining groups on the 20th day (Fig. 1).

\section{Evaluation of the disease}

\section{1) Clinical activity score}

UC was quantified with a clinical score assessing weight loss, stool consistency, and bleeding of the colon. No weight loss was counted as 0 points, weight loss of $1-5 \%$ as 1 point, $5-10 \%$ as 2 points, $10-20 \%$ as 3 points, and $20 \%$ or more as 4 points. For the stool consistency, the stool was collected in a thick paper and observed; 0 points were given for well-formed pellets, 2 points for pasty and semi-formed stools that did not stick to the anus, and 4 points for liquid stools that did stick to the anus. Bleeding was scored 0 points for no blood in the hem occult, 2 points for a positive hem occult, and 4 points for gross bleeding. The scores were added and divided by 3 , forming a total clinical score that ranged from 0.0 (healthy) to 4.0 (maximal activity of $\mathrm{UC}$ ).

\section{2) Gross lesion score}

An independent observer, who was blinded to the treatment, evaluated the severity of UC. For each animal, the distal $10 \mathrm{~cm}$ portion of the colon was removed and cut longitudi- 
nally, cleaned slightly in physiological saline to remove any fecal residues, and weighed. The macroscopic inflammation scores were assigned based on the clinical features of the colon using the following scoring pattern. No visible change was counted as 0 point, hyperemia at sites as 1 point, lesions with $>0$ to $1 \mathrm{~mm}$ diameter as 2 point, lesions with $>1$ to $2 \mathrm{~mm}$ diameter as 3 point, lesions with $>2$ to $5 \mathrm{~mm}$ diameter as 4 point, lesions with $>5$ to $10 \mathrm{~mm}$ diameter as 5 point, and lesions with $>10 \mathrm{~mm}$ diameter as 6 point. $^{13}$

\section{Histopathological study}

A $2 \mathrm{~mm}$ portion of the colonic specimen from each mouse was fixed in $10 \%$ formalin, embedded in paraffin, and cut into $5-\mu \mathrm{m}$ thick sections. The sections were stained with $\mathrm{H} \& \mathrm{E}$, and histopathological observations were made. The severity of colonic inflammation was scored using the four criteria, including submucosal edema, damage/necrosis, inflammatory cell infiltration, and vasculitis. Each of the criteria was graded on a scale from 0 to 3 , depending on the severity of the changes $(0=$ no change; $1=$ mild; $2=$ moderate; $3=$ severe $){ }^{13}$
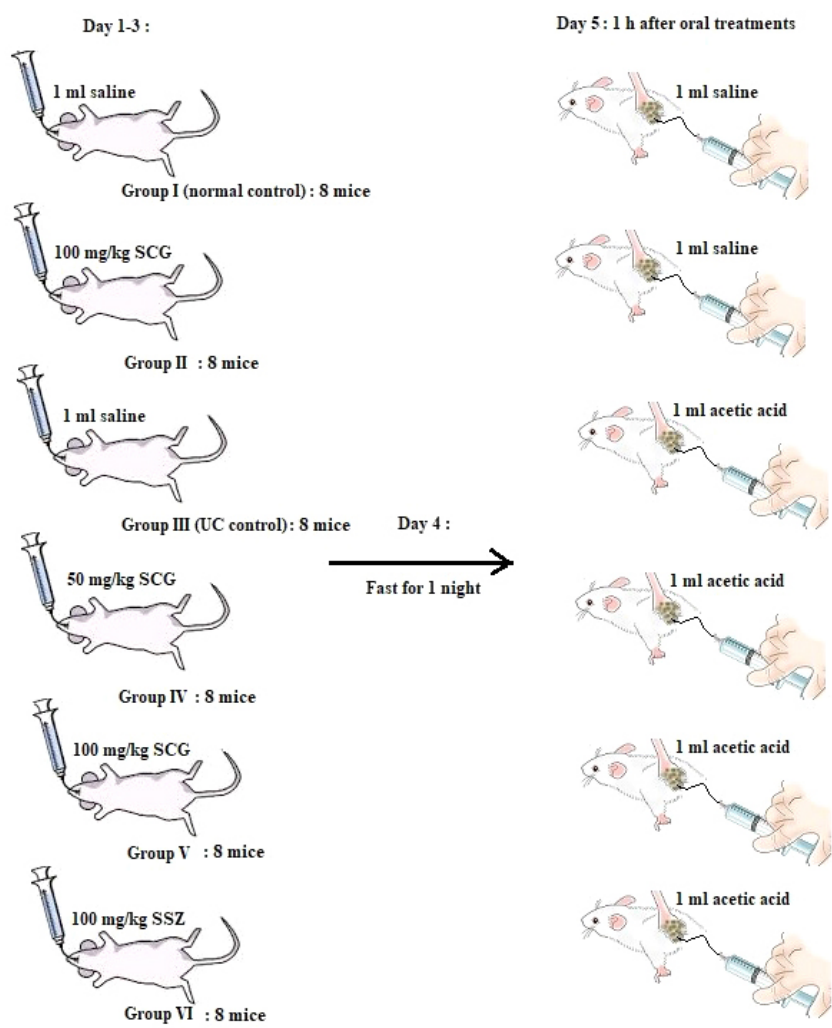

$1 \mathrm{ml}$ acetic acid

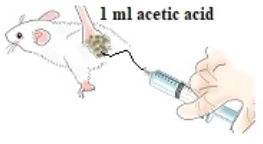

\section{Statistical methods}

Statistical analysis was conducted using one-way ANOVA with a Tukey test for multiple comparisons using SPSS version 16.0 (IBM Co., Armonk, NY, USA). The values are expressed as the mean \pm SEM of $n$ observations, where ' $n$ ' represents the number of rats ( $n=8$ animals for each group). $p$-values $<0.05$ were considered significant.

\section{RESULTS}

\section{Clinical activity and gross lesion scores}

In the AA-induced UC mice (group III), the clinical activity scores were significantly higher than those of the normal groups (I and II) $(3.21 \pm 0.31$ vs. $0.0 \pm 0.0, p<0.05)$ (Fig. 2). AA caused severe macroscopic edematous inflammation in the colon. In the treatment groups with 50 (group IV) and 100 (group V) mg/kg of SCG, the clinical activity score decreased to $2.67 \pm 0.18$ and $1.73 \pm 0.21$, $(p<0.05)$, respectively, compared to the UC control group (3.21 \pm 0.31$)$, and were higher than the standard treatment (group VI) of $100 \mathrm{mg} / \mathrm{kg} \mathrm{SSZ}$ (1.10 \pm 0.09$)$. In addition, the treatment groups with 50 and

Fig. 1. Schematic pattern of the experiments.

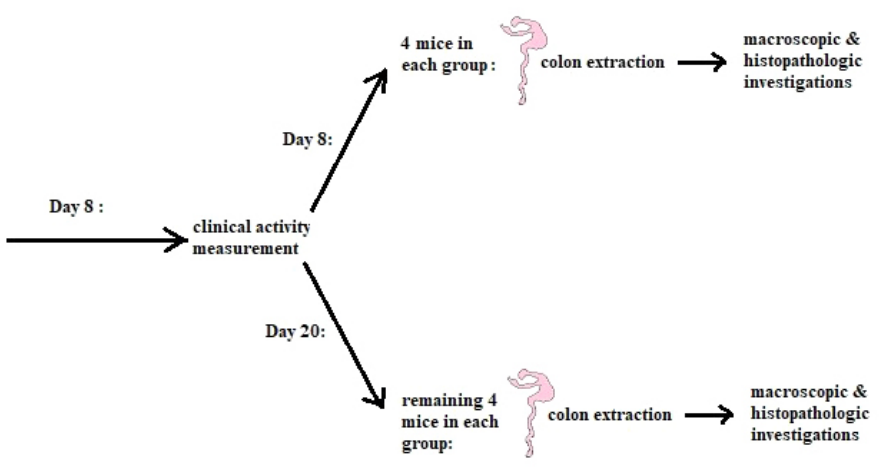




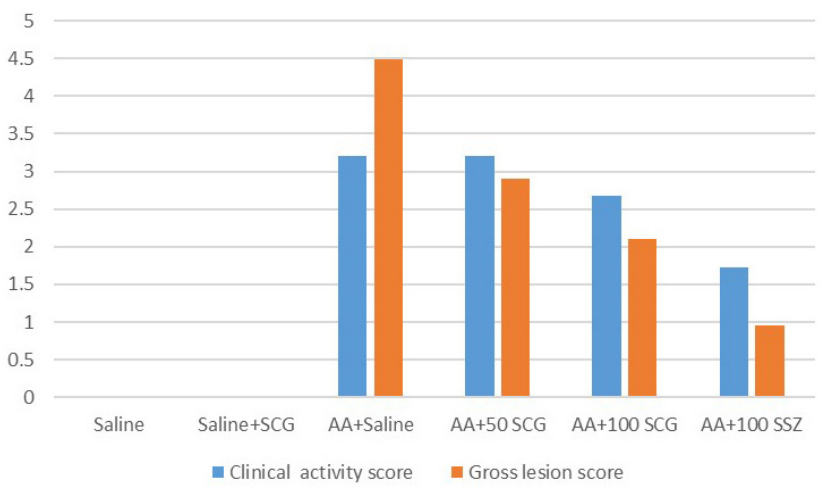

Fig. 2. Clinical activity and gross lesion score in the treatment groups. SCG, sodium cromoglycate; AA, acetic acid; SSZ, sulfasalazine.
$100 \mathrm{mg} / \mathrm{kg}$ of SCG showed a lower clinical gross lesion score than the UC control group $(2.91 \pm 0.28$ and $2.10 \pm 0.43$, vs. $4.49 \pm 0.61, p<0.05)$ but were higher than group $\mathrm{VI}$ $(0.95 \pm 0.18)$. On the 20th day, the clinical activity scores were not significantly different in all groups.

\section{Histopathological studies}

On the 8th day, the AA-induced UC control (group III) showed massive necrotic destruction of epithelium, sub-mucosal edema, areas of hemorrhages, and inflammatory cellular infiltration (Fig. 3C). The group IV treatment showed mini-
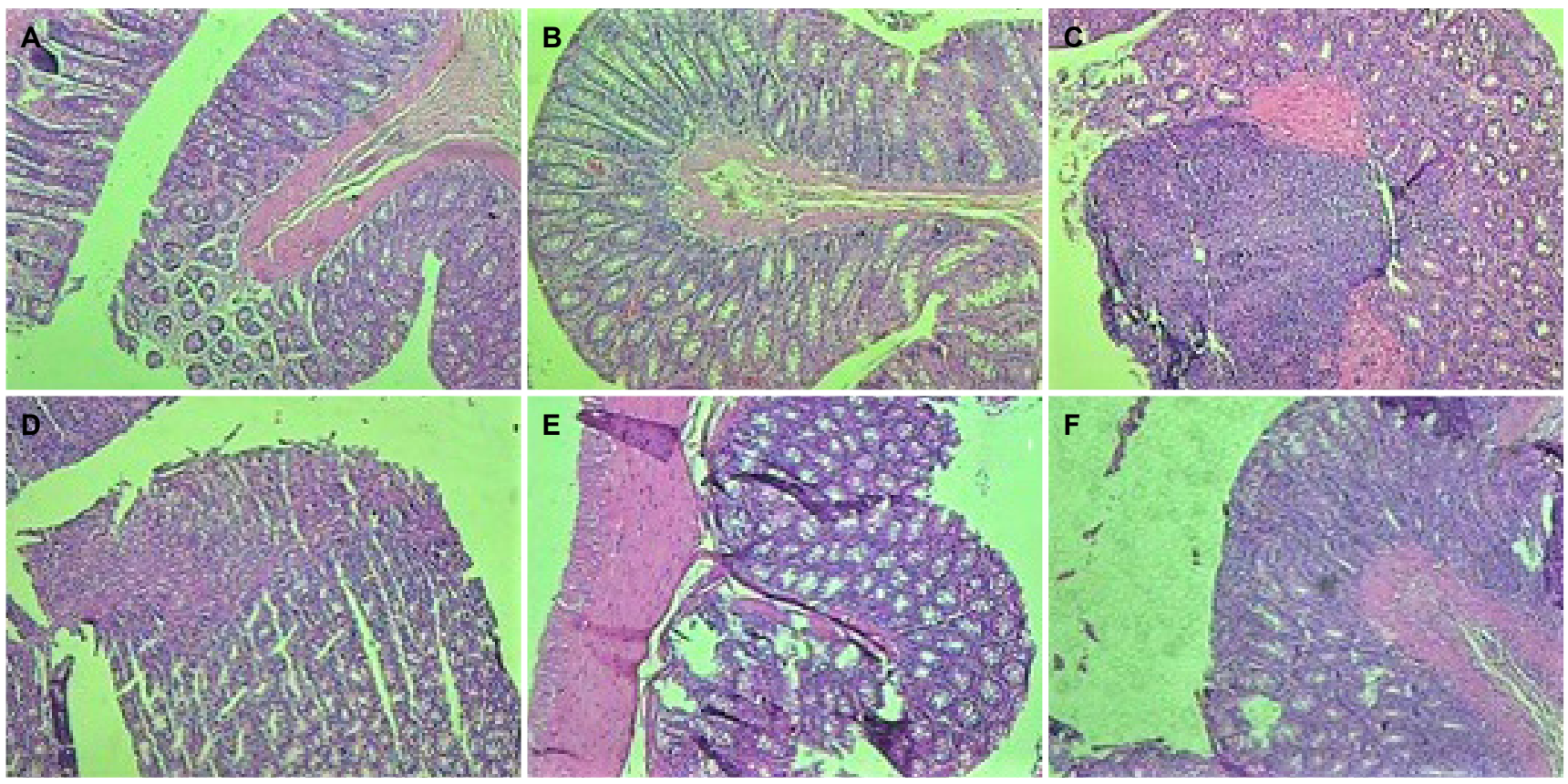

Fig. 3. Histopathological changes in the colon of the experimental mice. (A, B) Normal intact mucosa from groups I and II, showing an intact epithelial surface (H\&E, ×4). (C) Group III, acetic acid induced ulcerative colitis showing massive necrotic destruction of the epithelium, submucosal edema, areas of hemorrhage, and inflammatory cellular infiltration (H\&E, $\times 4$ ). (D) Group IV (SCG $50 \mathrm{mg} / \mathrm{kg}$ ) showing minimal damage to the mucosa with slight submucosal edema (H\&E, $\times 4$ ). (E) Group V (SCG $100 \mathrm{mg} / \mathrm{kg}$ ) showing significant protection of the colonic mucosa from acetic acid damage (H\&E, ×4). (F) Group VI (SSZ $100 \mathrm{mg} / \mathrm{kg}$ ) showing a normal colonic structure (H\&E, ×4). SCG, sodium cromoglycate; SSZ, sulfasalazine.

Table 1. Clinical Activity and Gross Lesion Features of Treatments in the Study

\begin{tabular}{lcc}
\hline Treatments & Clinical activity score & Gross lesion score \\
\hline Group I (saline) & $0.0 \pm 0.0$ & $0.0 \pm 0.0$ \\
Group II (saline+SCG) & $0.0 \pm 0.0$ & $0.0 \pm 0.0$ \\
Group III (AA+saline) & $3.21 \pm 0.31$ & $4.49 \pm 0.61$ \\
Group IV (AA+50 mg/kg SCG) & $2.67 \pm 0.18$ & $2.91 \pm 0.28$ \\
Group V (AA+100 mg/kg SCG) & $1.73 \pm 0.21$ & $2.10 \pm 0.43$ \\
Group VI (AA+100 mg/kg SSZ) & $1.10 \pm 0.09$ & $0.95 \pm 0.18$ \\
\hline
\end{tabular}

Values are presented as mean \pm standard error of the mean for groups of six animals each, Tukey-Kramer; values are statistically significant at $p<0.05$.

SCG, sodium cromoglycate; AA, acetic acid; SSZ, sulfasalazine. 
mal damage to the mucosa with slight sub-mucosal edema and mild inflammatory cell infiltration (Fig. 3D), while group $\mathrm{V}$ showed remarkable recovery of the colonic mucosa from the AA-induced UC damage (Fig. 3E). On the 20th day, tissue repair in groups IV, V, and III was higher than the control group, but the difference was not significant (Table 1).

\section{DISCUSSION}

UC-induced inflammation usually involves the colon or rectum, ${ }^{14}$ causing an atypical T-helper cell type 2 and T-helper cell type 9 response, which is recognized by the main symptoms of abdominal pain, diarrhea, and weight loss. ${ }^{15}$ Perioperative management of UC includes aminosalicylates (SSZ and mesalamine), glucocorticoid supplementation, purine analogues (6-mercaptopurine/azathioprine), cyclosporine, calcineurin inhibitors, methotrexate, and immunomodulator therapy with anti-tumor necrosis factor agents. ${ }^{16}$ For decades, SSZ, which had been categorized under aminosalicylates, was used as the first-line medication for UC. SSZ is absorbed in the small intestine. After re-excreting in the bile, the drug is transferred to the colon, having its azo bond split by bacteria and producing sulfapyridine and 5-aminosalicylic acid. Sulfapyridine, which is the main cause of side effects, is then absorbed. ${ }^{17}$ The drug's side effects are frequent but mostly reversible because of the sulfapyridine concentrations and low acetylation of the drug, some of which include gastrointestinal intolerance, malaise, headache, arthralgia, drug fever, red blood cells disorders, reversible hypospermia, and in rare cases, neurotoxicity, hepatotoxicity, poly-arteritis, and pulmonary fibrosis. ${ }^{18}$ In one case, SSZ-induced pancytopenia was detected due to bone marrow suppression. According to that report, the treatment with SSZ needed to be ceased, and an urgent alternative therapy was needed for the patient. ${ }^{19}$ Many other studies were in agreement with the urgency of a substitute or concurrent treatment in conjunction with SSZ. ${ }^{20}$ According to the data published by Shi et al. ${ }^{21}$ combination therapy of SSZ and bifidobacteria for a UC treatment is more effective in inhibiting the inflammatory processes and regulating immune systems.

According to the literature regarding SCG, SCG administration is suitable for long-term UC management, as well as for improving the patients' sense of well-being, and the sigmoidoscopic and rectal biopsy results. ${ }^{9,22}$ The data show that SCG is an effective treatment for chronic or recurrent enterocolitis in patients with Hirschsprung's disease. This is because SCG is not absorbed intestinally and there are no systemic side effects in its application. ${ }^{23}$ The equipotent effect of SCG on the pylorus ligation model provides strong evidence for the antiulcer activity of the drug. This was based on the significant increase in the mucin content and the decrease in the total protein and pepsin contents in rats treated with $\mathrm{SCG}^{24}$ In the present study, the clinical and macroscopic characteristics of SCG-treated AA-induced UC mice were compared with a SSZ-treated group as a standard treatment. On 8th day, the group IV mice, treated with $50 \mathrm{mg} / \mathrm{kg}$ SCG, showed minimal mucosa damage with slight submucosal edema. At a higher dose of $100 \mathrm{mg} / \mathrm{kg}$ SCG (group V), significant protection of the colonic mucosa was detected from AA-induced UC damage.

On the other hand, no significant difference was observed between the SSZ and SCG-treated groups compared to the control group on the 20th day, which suggests that the test is not appropriate for a long time. As the animal model of UC shows the acute form of the disease and includes the exacerbation of symptoms as well as recovery from the clinical symptoms after a few days, there was no histopathological difference between the treated groups and controls on the 20th day. Indeed, the disease does not become chronic after this period. These results are equivalent to those of a study that assessed the anti-ulcer effect of SCG on non-steroidal anti-inflammatory drugs, and ethanol-induced ulcers in rats, declaring the effectiveness of SCG in healing ulcers. ${ }^{9}$ The SSZ-treated group in this study (group $\mathrm{VI}$ ), showed a normal colonic structure. According to the results, the potency of SCG to reduce the gross lesion score was far more than its ability to reduce the clinical activity score, and this potency was accelerated at higher SCG doses (Fig. 2). This evidence was correlated with the histopathological manifestations in all treatments in the study (Fig. 3).

$\mathrm{UC}$ is a chronic disease that takes a long time (perhaps many years) to develop, including the onset of its clinical manifestations and symptoms. ${ }^{25,26}$ In the UC model in this study, UC caused by AA developed in less than a month. Therefore, it is related more closely to the acute form of IBD or UC, which manifests symptoms similar to exacerbation or inflammation in UC-induced mice. ${ }^{26-28}$ SCG is well known for its mast cell stabilizer properties; ${ }^{29}$ it inhibits the release of 
histamine, leukotrienes, and slow-reacting substance of anaphylaxis from mast cells by inhibiting degranulation after exposure to reactive antigens. ${ }^{30,31}$ According to the results and data from this study, SCG could produce a palliative and partial recovery effect in the treatment groups compared to the control group.

In conclusion, the present data suggest that the SCG treatment of mice with a dose-response procedure effectively prevents AA-induced UC in mice. This protective effect highlights the alternative role of SCG as the standard treatment of UC.

\section{REFERENCES}

1. Fakhoury M, Negrulj R, Mooranian A, Al-Salami H. Inflammatory bowel disease: clinical aspects and treatments. J Inflamm Res 2014;7:113-120.

2. Li J, Ueno A, Fort Gasia M, et al. Profiles of lamina propria T helper cell subsets discriminate between ulcerative colitis and Crohn's disease. Inflamm Bowel Dis 2016;22:1779-1792.

3. Shivashankar R, Lichtenstein GR. Mimics of inflammatory bowel disease. Inflamm Bowel Dis 2018;24:2315-2321.

4. Asakura H, Kitahora T. Antioxidants and polyphenols in inflammatory bowel disease: ulcerative colitis and Crohn disease. In: Watson RR, Preedy VR, Zibadi S, eds. Polyphenols: prevention and treatment of human disease. Volume 2. 2nd ed. Cambridge (MA): Academic Press, 2018:279-292.

5. Stasikowska-Kanicka O, Danilewicz M, Głowacka A, Wągrowska-Danilewicz M. Mast cells and eosinophils are involved in activation of ulcerative colitis. Adv Med Sci 2012;57:230-236.

6. Wirtz S, Popp V, Kindermann M, et al. Chemically induced mouse models of acute and chronic intestinal inflammation. Nat Protoc 2017;12:1295-1309.

7. Bonovas S, Lytras T, Nikolopoulos G, Peyrin-Biroulet L, Danese S. Systematic review with network meta-analysis: comparative assessment of tofacitinib and biological therapies for moderate-to-severe ulcerative colitis. Aliment Pharmacol Ther 2018; 47:454-465.

8. Taylor K, Gibson PR. Conventional therapy of ulcerative colitis: corticosteroids. In: Baumgart DC, ed. Crohn's disease and ulcerative colitis. 2nd ed. Melbourne: Springer, 2017:399-412.

9. Sravani MR, Naveen A. Anti-ulcer effect of sodium cromoglycate in NSAID and ethanol induced ulcer in comparison with ranitidine in rats. Pharma Innov 2017;6:1-5.

10. Roviezzo F, Sorrentino R, lacono VM, et al. Disodium cromoglycate inhibits asthma-like features induced by sphingosine-1-phosphate. Pharmacol Res 2016;113(Pt A):626-635.

11. Bischoff SC. Mast cells in gastrointestinal disorders. Eur J Pharmacol 2016;778:139-145.

12. Lieberman JA, Zhang J, Whitworth J, Cavender C. A randomized, double-blinded, placebo-controlled study of the use of viscous oral cromolyn sodium for the treatment of eosinophilic esophagitis. Ann Allergy Asthma Immunol 2018;120:527-531.

13. Cohen RD. Inflammatory bowel disease: diagnosis and therapeutics. 1st ed. Totowa (NJ): Humana Press, 2003

14. Henriksen M, Høivik ML, Jelsness-Jørgensen LP, Moum B, IBSEN Study Group. Irritable bowel-like symptoms in ulcerative colitis are as common in patients in deep remission as in inflammation: results from a population-based study [the IBSEN study]. J Crohns Colitis 2018;12:389-393.

15. Khan I, Samson SE, Grover AK. Antioxidant supplements and gastrointestinal diseases: a critical appraisal. Med Princ Pract 2017;26:201-217.

16. Vasudevan A, Gibson PR, van Langenberg DR. Time to clinical response and remission for therapeutics in inflammatory bowel diseases: what should the clinician expect, what should patients be told? World J Gastroenterol 2017;23:6385-6402.

17. Kappelman MD, Rifas-Shiman SL, Porter CQ, et al. Direct health care costs of Crohn's disease and ulcerative colitis in US children and adults. Gastroenterology 2008;135:1907-1913.

18. Kotze PG, Albuquerque IC, Moraes AC, Vieira A, Souza F. Análise de custo-minimização entre o infliximabe (IFX) e o adalimumabe (ADA) no tratamento da doença de Crohn (DC). Rev Bras Coloproct 2009;29:158-168.

19. Shailubhai K, Palejwala V, Arjunan KP, et al. Plecanatide and dolcanatide, novel guanylate cyclase-C agonists, ameliorate gastrointestinal inflammation in experimental models of murine colitis. World J Gastrointest Pharmacol Ther 2015;6:213-222.

20. Solberg IC, Lygren I, Jahnsen J, et al. Clinical course during the first 10 years of ulcerative colitis: results from a population-based inception cohort (IBSEN study). Scand J Gastroenterol 2009; 44:431-440.

21. Shi Y, Liu H, Huang Z, Liu Z. Clinical effects of sulfasalazine combined with live combined bifidobacterium lactobacillus and enterococcus capsules in patients with ulcerative colitis. Chinese Journal of New Drugs and Clinical Remedies 2010;10:783-785.

22. Shin MR, Kim KJ, Kim SH, et al. Comparative evaluation between sulfasalazine alone and in combination with herbal medicine on DSS-induced ulcerative colitis mice. Biomed Res Int 2017;2017: 6742652.

23. Rintala RJ, Lindahl H. Sodium cromoglycate in the management of chronic or recurrent enterocolitis in patients with Hirschsprung's disease. J Pediatr Surg 2001;36:1032-1035.

24. Srivastava V, Viswanathaswamy AH, Mohan G. Determination of the antiulcer properties of sodium cromoglycate in pylorus-ligated albino rats. Indian J Pharmacol 2010;42:185-188.

25. Doherty GA, Cheifetz AS. Management of acute severe ulcerative colitis. Expert Rev Gastroenterol Hepatol 2009;3:395-405.

26. Jiminez JA, Uwiera TC, Douglas Inglis G, Uwiera RR. Animal models to study acute and chronic intestinal inflammation in mammals. Gut Pathog 2015;7:29.

27. Kopecki Z, Yang G, Treloar S, et al. Flightless I exacerbation of inflammatory responses contributes to increased colonic damage in a mouse model of dextran sulphate sodium-induced ulcerative colitis. Sci Rep 2019;9:12792.

28. Yokooji T, Matsuo H. Sodium cromoglycate prevents exacerbation of IgE-mediated food-allergic reaction induced by aspirin in a rat model of egg allergy. Int Arch Allergy Immunol 2015; 167:193-202.

29. Stoyanova II, Gulubova MV. Mast cells and inflammatory media- 
tors in chronic ulcerative colitis. Acta Histochem 2002;104: 185-192.

30. Jiang L, Fang P, Septer S, Apte U, Pritchard MT. Inhibition of mast cell degranulation with cromolyn sodium exhibits organ-specific effects in polycystic kidney (PCK) rats. Int J Toxicol 2018;37:
308-326.

31. Oka T, Kalesnikoff J, Starkl P, Tsai M, Galli SJ. Evidence questioning cromolyn's effectiveness and selectivity as a 'mast cell stabilizer' in mice. Lab Invest 2012;92:1472-1482. 